\title{
Classifying hydrogen-rich superconductors
}

\author{
E. F. Talantsev ${ }^{1,2}$ \\ ${ }^{1}$ M.N. Miheev Institute of Metal Physics, Ural Branch, Russian Academy of Sciences, \\ 18, S. Kovalevskoy St., Ekaterinburg, 620108, Russia \\ ${ }^{2}$ NANOTECH Centre, Ural Federal University, 19 Mira St., Ekaterinburg, 620002, \\ Russia
}

\section{E-mail: evgeny.talantsev@imp.uran.ru}

\begin{abstract}
The era of near-room-temperature superconductivity started after experimental discovery by Drozdov et al (2015 Nature $\mathbf{5 2 5}$ 73) who found that compressed $\mathrm{H}_{3} \mathrm{~S}$ exhibits superconducting transition at $T_{\mathrm{c}}=203 \mathrm{~K}$. To date, the record near-room-temperature superconductivity stands with another hydrogen-rich highly compressed compound, $\mathrm{LaH}_{10}$ (Somayazulu et al 2019 Phys. Rev. Lett. 122 027001), which has critical temperature of $T_{c}>$ $240 K$. In this paper, we analyse available upper critical field, $B_{\mathrm{c} 2}(T)$, data for $\mathrm{LaH}_{10}$ (Drozdov et al 2019 Nature $\mathbf{5 6 9}$ 528) and report that this compound in all considered scenarios has the ratio of $T_{\mathrm{c}}$ to the Fermi temperature, $T_{\mathrm{F}}, 0.009<T_{\mathrm{c}} / T_{\mathrm{F}}<0.038$, which is typical range for unconventional superconductors. In attempt to extend our finding, we examined experimental $B_{\mathrm{c} 2}(T)$ data for superconductors in the palladium-hydrogen $\left(\mathrm{PdH}_{\mathrm{x}}\right)$ and thorium-hydrogen-deiterium (ThH-ThD) systems and surprisingly find that superconductors in both these systems also fall into unconventional superconductors band. Taking in account that $\mathrm{H}_{3} \mathrm{~S}$ has the ratio of $0.012<T_{\mathrm{c}} / T_{\mathrm{F}}<0.039$ (Talantsev 2019 Mod. Phys. Lett. B 33 1950195) we come to conclusion that in the Uemura plot all discovered to date hydrogen-rich superconductors, i.e. $\mathrm{Th}_{4} \mathrm{H}_{15}-\mathrm{Th}_{4} \mathrm{D}_{15}, \mathrm{PdH}_{\mathrm{x}}, \mathrm{H}_{3} \mathrm{~S}$ and $\mathrm{LaH}_{10}$ (in this list we do not include $\mathrm{NbTiH}_{\mathrm{x}}, \mathrm{PtH}_{\mathrm{x}}, \mathrm{SiH}_{4}$, and $\mathrm{PH}_{3}$ for which experimental data beyond $T_{\mathrm{c}}$ are unknown), lie in same band as all unconventional superconductors, particularly heavy
\end{abstract}


fermions, fullerenes, pnictides, and cuprates, and former should be classified as a new class of unconventional superconductors.

\section{Introduction}

The discovery of superconductivity in highly compressed $\mathrm{H}_{3} \mathrm{~S}$ with $T_{\mathrm{c}}=203 \mathrm{~K}$ by Drozdov et al [1] is the most fascinating breakthrough in superconductivity since epochal discovery of high-temperature superconductivity in cuprates by Bednoltz and Mueller [2]. Historical aspects of the problem can be found elsewhere [3-6].

Despite a fact that $\mathrm{H}_{3} \mathrm{~S}$ is widely classified as conventional [7] electron-phonon superconductor [1,3-6,8-13], we performed [14] analysis of experimental upper critical field data, $B_{\mathrm{c} 2}(T)$, reported by Mozaffari et al $[15,16]$, and showed that $\mathrm{H}_{3} \mathrm{~S}$ is unconventional superconductor which lies in common unconventional superconductors trend band of the Uemura plot $[17,18]$ together with other unconventional superconductors, i.e. heavy fermions, fullerenes, pnictides, and cuprates.

The concept of Uemura plot [18] is to represent all 32 classes of superconductors [19] by using two fundamental temperatures: one is the superconducting transition temperature, $T_{\mathrm{c}}$ (usually, this is used as Y-axis), and another is the Fermi temperature, $T_{\mathrm{F}}$ (usually, this is used as $\mathrm{X}$-axis) [18]. In this representation all known unconventional superconductors fill a narrow band of $10^{-2}<T_{c} / T_{F}<5 \cdot 10^{-2}$, while BCS electron-phonon mediated superconductors are located in the area of $T_{c} / T_{F}<4 \cdot 10^{-4}$. We showed [14] that $\mathrm{H}_{3} \mathrm{~S}$ compressed at $P=150 \mathrm{GPa}$ and $155 \mathrm{GPa}$ has $T_{\mathrm{c}} / T_{\mathrm{F}}$ ratio in the interval of

$1.2 \cdot 10^{-2}<T_{c} / T_{F}<3.9 \cdot 10^{-2}$, and, thus, based on general approach of Uemura et al $[17,18], \mathrm{H}_{3} \mathrm{~S}$ should be classified as unconventional superconductor.

Recently, further step towards room-temperature superconductivity was made by Somayazulu et al [20] who discovered near-room-temperature superconductivity in another 
highly-compressed hydrogen-rich compound of $\mathrm{LaH}_{10}$. The latter exhibits transition temperature of $T_{C} \gtrsim 240 \mathrm{~K}$ at external pressure in the range of $P=150-200 \mathrm{GPa}[20,21]$.

Drozdov et al [21] reported experimental upper critical field data for $\mathrm{LaH}_{10}$ which we analyse in this paper and find that in all considered scenarios this compound has the ratio of superconducting energy gap, $\Delta(0)$, to the Fermi energy, $\varepsilon_{\mathrm{F}}$, of $0.02<\Delta(0) / \varepsilon_{\mathrm{F}}<0.07$, with respective ratio of $T_{\mathrm{c}}$ to the Fermi temperature, $T_{\mathrm{F}}, 0.009<T_{\mathrm{c}} / T_{\mathrm{F}}<0.038$. As the result, in Uemura plot $\mathrm{LaH}_{10}$ lies in the same band as all unconventional superconductors and falls just above another hydrogen-rich counterpart of $\mathrm{H}_{3} \mathrm{~S}$ [14].

To prove that our findings in regard of $\mathrm{H}_{3} \mathrm{~S}$ and $\mathrm{LaH}_{10}$ are generic features of hydrogenrich superconductors we re-examine the upper critical field data for the first discovered superhydride superconductor $\mathrm{Th}_{4} \mathrm{H}_{15}$ (by Satterthwaite and Toepke [22] in 1970), and, perhaps, the most experimentally studied to date hydrogen-rich superconductors in palladium-hydrogen-deuterium system, $\mathrm{PdH}-\mathrm{PhD}$, discovered by Skoskiewicz [23] and show that despite a fact that thorium-hydrogen-deuterium and palladium-hydrogen compounds have $T_{\mathrm{c}}<8.5 \mathrm{~K}$, these low-temperature superconductors have similar ratios of $\Delta(0) / \varepsilon_{\mathrm{F}}$ and $T_{\mathrm{c}} / T_{\mathrm{F}}$ as ones of its near-room-temperature superconducting counterparts.

We should note that the silane, $\mathrm{SiH}_{4}$, was the first discovered by Eremets group highlycompressed hydrogen-rich superconductor with $T_{c}=17 \mathrm{~K}$ (observed at pressure of $P=96$ $120 \mathrm{GPa}$ ) [24]. Covalent hydride phosphine, $\mathrm{PH}_{3}$, is another hydrogen-rich superconductor in which superconductivity with $T_{c} \simeq 100 \mathrm{~K}$ was discovered at $P \gtrsim 200 \mathrm{GPa}$ [25]. And recently, superconductivity in $\operatorname{PtH}_{x}(x \cong 1)$ was discovered experimentally at $P=30 \mathrm{GPa}$ [26]. $\mathrm{NbTiH}_{x}[27]$ is another hydrogen-rich superconductor which can be potentially considered. Unfortunately, for all of these compounds, fundamental experimental data beyond $T_{\mathrm{c}}$ are unknown, and, thus, we were not able to analyse these materials in our consideration herein. 
In result, we show that all discovered to date hydrogen-rich superconductors for which experimental data beyond $T_{\mathrm{c}}$ are available, i.e. $\mathrm{Th}_{4} \mathrm{H}_{15}-\mathrm{Th}_{4} \mathrm{D}_{15}, \mathrm{PdH}_{\mathrm{x}}, \mathrm{H}_{3} \mathrm{~S}$ and $\mathrm{LaH}_{10}$, lie in the same band in the Uemura plot as all unconventional superconductors (particularly heavy fermions, fullerenes, pnictides, and cuprates) and thus, these superconductors should be classified as a new family of unconventional superconductors.

\section{The upper critical field models}

Ground state upper critical field, $B_{\mathrm{c} 2}(0)$, in the Ginzburg-Landau theory [28] is given by:

$$
B_{c 2}\left(\frac{T}{T_{c}}=0\right)=\frac{\phi_{0}}{2 \cdot \pi \cdot \xi^{2}(0)}
$$

where $\phi_{0}=2.068 \cdot 10^{-15} \mathrm{~Wb}$ is magnetic flux quantum, and $\xi(0)$ is the ground state coherence length. For the temperature dependent upper critical field data one of the most rigours model was proposed by Werthamer, Helfand, and Hohenberg (WHH) [29,30]:

$$
\ln \left(\frac{T}{T_{C}(B=0)}\right)=\psi\left(\frac{1}{2}\right)-\psi\left(\frac{1}{2}+\frac{\hbar \cdot D \cdot B_{C 2}(T)}{2 \cdot \phi_{0} \cdot k_{B} \cdot T}\right)
$$

where $\psi$ is digamma function, $D$ is the diffusion constant of the normal conducting electrons/holes. However, application of this model for the analysis of experimental $B_{\mathrm{c} 2}(T)$ data requires very often and uniformly measured dataset which covers the whole temperature range of $0<T<T_{c}$ with approximate step of $(0.02-0.05) \cdot T_{c}$ between data points. In real world experiments, this condition is usually impossible to achieve due to either experimental limitation to cool sample down to low enough reduced temperatures, $T / T_{\mathrm{c}}$, either to create reasonably high applied magnetic field, $B_{\mathrm{appl}} / B_{\mathrm{c} 2}(0)$, or, in most cases, the both.

Based on this, the analysis of $B_{\mathrm{c} 2}(T)$ data, as a rule, performs by the utilization of other approaches, different from WHH [29,30], which were developed for real world experiments, i.e. when $B_{\mathrm{c} 2}(T)$ dataset is limited by measurements performed at high reduced temperatures, $\frac{1}{2} \lesssim \frac{T}{T_{c}}$. We stress that this is entire case for highly compressed $\mathrm{LaH}_{10}$ for which $B_{\mathrm{c} 2}(T)$ data is 
available to date only in narrow temperature range of $0.96 \leq \frac{T}{T_{c}} \leq 1.0$ [21]. Primary reason for this is that available in experiment applied magnetic field was limited by $B_{a p p l} \leq 9 \mathrm{~T}$ [21], which is typical for modern labs (for instance, conventional PPMS systems provide this). However, if even world top quasi-DC magnetic field facility will be in use $[15,16,31]$ and maximum applied magnetic field of $B_{a p p l}=62-65 T$ will be utilized, then based on estimated value for $B_{\mathrm{c} 2}(0)=90-140 \mathrm{~T}$ (given by Drozdov et al [21]) for $\mathrm{LaH}_{10}$, it is unlikely that $B_{\mathrm{c} 2}(T)$ measurements will be possible to perform at reduced temperature lower than $0.7 \leq \frac{T}{T_{c}}$, i.e., $170 K \leq T$.

The reality is that robust use of WHH model $[29,30]$ for highly compressed $\mathrm{LaH}_{10}$ (and, probably, for others homological compounds of $\mathrm{La}_{n} \mathrm{H}_{m}$ series [32,33]) will be unlikely in near decades. Thus, different models need to be used to analyse experimental $B_{\mathrm{c} 2}(T)$ data for nearroom-temperature superconductors. One of the approach, which was actually proposed by Werthamer, Helfand, and Hohenberg [29,30], is extrapolative expression:

$$
B_{c 2}(0)=\frac{\phi_{0}}{2 \cdot \pi \cdot \xi^{2}(0)}=-0.693 \cdot T_{C} \cdot\left(\frac{d B_{c 2}(T)}{d T}\right)_{T \sim T_{C}}
$$

In this paper, we will designate Eq. 3 as WHH model.

Another model, which is also based on Werthamer, Helfand, and Hohenberg primary idea $[29,30]$, but one accurately generates full $B_{\mathrm{c} 2}(T)$ extrapolative curve from experimental data measured at high reduced temperatures, $T / T_{\mathrm{c}}$, was developed by Baumgartner et al [34]:

$$
\begin{aligned}
& B_{c 2}(T)=B_{c 2}(0) \cdot\left(\frac{\left(1-\frac{T}{T_{C}}\right)-0.153 \cdot\left(1-\frac{T}{T_{C}}\right)^{2}-0.152 \cdot\left(1-\frac{T}{T_{C}}\right)^{4}}{0.693}\right)= \\
& =\frac{\phi_{0}}{2 \cdot \pi \cdot \xi^{2}(0)} \cdot\left(\frac{\left(1-\frac{T}{T_{C}}\right)-0.153 \cdot\left(1-\frac{T}{T_{C}}\right)^{2}-0.152 \cdot\left(1-\frac{T}{T_{C}}\right)^{4}}{0.693}\right)
\end{aligned}
$$

We will designate this model as B-WHH model.

In addition, we will use classical two-fluid Gorter-Casimir model (GC model) [35,36]: 


$$
B_{c 2}(T)=B_{c 2}(0) \cdot\left(1-\left(\frac{T}{T_{c}}\right)^{2}\right)=\frac{\phi_{0}}{2 \cdot \pi \cdot \xi^{2}(0)} \cdot\left(1-\left(\frac{T}{T_{c}}\right)^{2}\right)
$$

which is in a wide use too $[1,15,21,37,38]$. We note, that Drozdov et al [1], Mozaffari et al $[15,16]$ and Drozdov et al [21] designate Eq. 5 as the Ginzburg-Landau theory equation [28]. This is incorrect, because the latter is [28,35]:

$$
B_{c 2}(T)=B_{c 2}(0) \cdot\left(1-\left(\frac{T}{T_{c}}\right)\right)=\frac{\phi_{0}}{2 \cdot \pi \cdot \xi^{2}(0)} \cdot\left(1-\left(\frac{T}{T_{c}}\right)\right)
$$

There is also $B_{\mathrm{c} 2}(T)$ model proposed by Gor'kov [39] which was written in analytical form by Jones et al. [40]:

$$
\begin{aligned}
& B_{c 2}(T)=B_{c 2}(0) \cdot\left(\frac{1.77-0.43 \cdot\left(\frac{T}{T_{C}}\right)^{2}+0.07 \cdot\left(\frac{T}{T_{C}}\right)^{4}}{1.77}\right) \cdot\left[1-\left(\frac{T}{T_{C}}\right)^{2}\right]= \\
& \frac{\phi_{0}}{2 \cdot \pi \cdot \xi^{2}(0)} \cdot\left(\frac{1.77-0.43 \cdot\left(\frac{T}{T_{c}}\right)^{2}+0.07 \cdot\left(\frac{T}{T_{C}}\right)^{4}}{1.77}\right) \cdot\left[1-\left(\frac{T}{T_{C}}\right)^{2}\right]
\end{aligned}
$$

\section{III. $\mathrm{Th}_{4} \mathrm{H}_{15}$ - $\mathrm{Th}_{4} \mathrm{D}_{15}$ superconductors in Uemura plot}

We start our consideration with the first discovered superhydride superconductors, i.e., $\mathrm{Th}_{4} \mathrm{H}_{15}$ and $\mathrm{Th}_{4} \mathrm{D}_{15}[22]$. We should note, that already in the first paper of 1970 , Satterthwaite and Toepke [22] reported the absence of the isotope effect in ThH-ThD system:

$$
T_{c} \cdot M^{\alpha}=\text { const }
$$

where $M$ is isotope mass, and $\alpha \approx 1 / 2$ for weak-coupling limit of BCS theory [7], which is one of indispensable fundamental feature of electron-phonon mediated superconductivity [7]. Later, Stritzker and Buckel [41] experimentally found that the isotope effect in the palladiumhydrogen-deuterium (PdH-PdD) system has opposite sign (so called, reverse isotope effect). Yussouff et al. [42] extended this discovery on palladium-hydrogen-deuterium-tritium system (PdH-PdD-PdT). This reverse isotope effect in PdH-PdD-PdT system is still under wide discussion $[43,44]$. In regard of ThH-ThD system, detailed studied by Caton and 
Satterthwaite [45] showed that superconductors in thorium-hydrogen-deuterium (ThH-ThD) system have also reverse isotope effect.

From the author knowledge, available to date experimental data for the upper critical field, $B_{\mathrm{c} 2}(T)$, are limited by values reported by Satterthwaite and Toepke [22]. The authors reported for both, $\mathrm{Th}_{4} \mathrm{H}_{15}$ and $\mathrm{Th}_{4} \mathrm{D}_{15}$, compounds:

$$
B_{c 2}(T \sim 0)=2.5-3.0 T
$$

From these values, the ground state coherence length, $\xi(0)$, for $\mathrm{Th}_{4} \mathrm{H}_{15}$ and $\mathrm{Th}_{4} \mathrm{D}_{15}$ phases, can be derived as following:

$$
\xi(0)=11.0 \pm 0.5 \mathrm{~nm}
$$

Miller et al. [46] for both phases reported the BCS ratio within a range:

$$
\alpha=\frac{2 \cdot \Delta(0)}{k_{B} \cdot T_{c}}=3.42-3.47
$$

By utilizing superconducting transition temperature for $\mathrm{Th}_{4} \mathrm{H}_{15}$ and $\mathrm{Th}_{4} \mathrm{D}_{15}$ phases [22]:

$$
T_{c}=8.20 \pm 0.15 \mathrm{~K}
$$

one can deduce ground state superconducting energy gap:

$$
\Delta(0)=1.22 \pm 0.03 \mathrm{meV}
$$

and by using well-known BCS expression [7]:

$$
\xi(0)=\frac{\hbar \cdot v_{F}}{\pi \cdot \Delta(0)}
$$

where $\hbar=h / 2 \pi$ is reduced Planck constant, one can calculate the Fermi velocity, $v_{\mathrm{F}}$, in $\mathrm{Th}_{4} \mathrm{H}_{15}$ and $\mathrm{Th}_{4} \mathrm{D}_{15}$ phases:

$$
v_{F}=\pi \cdot \frac{\xi(0) \cdot \Delta(0)}{\hbar}=(6.4 \pm 0.2) \cdot 10^{4} \mathrm{~m} / \mathrm{s}
$$

To place $\mathrm{Th}_{4} \mathrm{H}_{15}$ and $\mathrm{Th}_{4} \mathrm{D}_{15}$ phases in the Uemura plot [18], we need to make assumption about the effective charge carrier mass, $m_{e f f}^{*}$, to calculate the Fermi temperature, $T_{\mathrm{F}}$ :

$$
T_{F}=\frac{\varepsilon_{F}}{k_{B}}=\frac{m_{e f f}^{*} \cdot v_{F}^{2}}{2 \cdot k_{B}}
$$


Due to there is no any available experimental values to date for $\mathrm{Th}_{4} \mathrm{H}_{15}$ and $\mathrm{Th}_{4} \mathrm{D}_{15}$ phases we used the simplest assumption:

$$
m_{e f f}^{*}=1.0 \cdot m_{e}
$$

and one can calculate the Fermi temperature, $T_{\mathrm{F}}$ :

$$
T_{F}=\frac{\varepsilon_{F}}{k_{B}}=\frac{m_{e f f}^{*} \cdot v_{F}^{2}}{2 \cdot k_{B}}=137 \pm 9 K
$$

and the ratio:

$$
\frac{T_{C}}{T_{F}}=0.060 \pm 0.005
$$

which places both $\mathrm{Th}_{4} \mathrm{H}_{15}$ and $\mathrm{Th}_{4} \mathrm{D}_{15}$ phases in the Uemura plot in the upper boarder of the unconventional superconductor (Fig. 1).

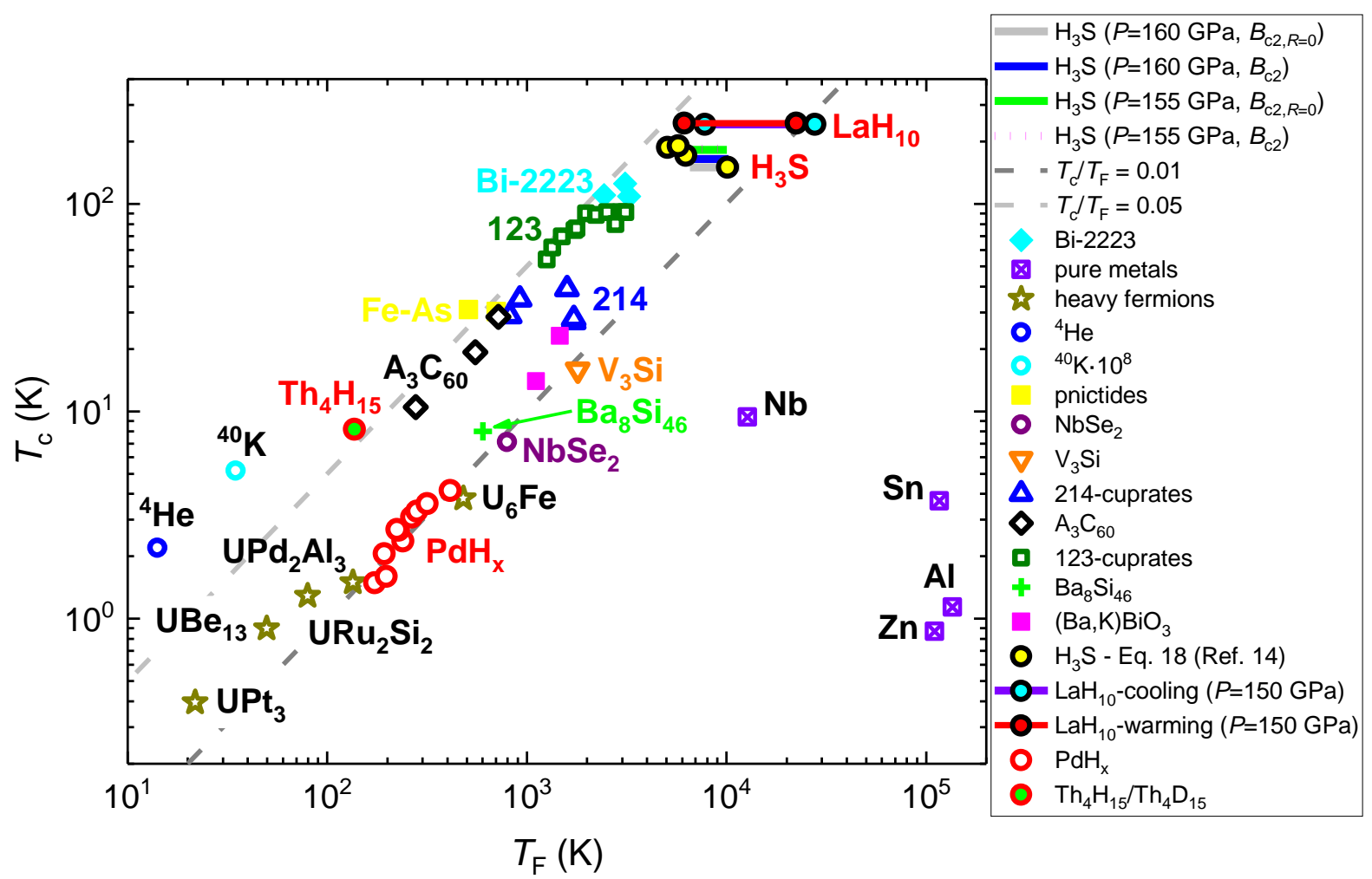

Figure 1. A plot of $T_{\mathrm{c}}$ versus $T_{\mathrm{F}}$ obtained for most representative superconducting families including $\mathrm{PdH}_{\mathrm{x}}, \mathrm{H}_{3} \mathrm{~S}$ and $\mathrm{LaH}_{10}$. Data was taken from Uemura [18], Ye et al. [47], Qian et al. [48], Hashimoto et al. [49] and Ref. 14. 


\section{PdH $_{x}$ Superconductors in Uemura plot}

Surprisingly enough, to author's knowledge, as thorium-hydrogen-deuterium superconductors discovered by Satterthwaite and Toepke [22] in 1970, as palladiumhydrogen-deuterium superconductors discovered by Skoskiewicz [23] in 1973 have never been located in the Uemura plot [18]. To do this, we utilize results of systematic studies of the upper critical field for palladium-hydrogen superconductors reported by Balbaa and Manchester [50]. We show their results (i.e., $T_{\mathrm{c}}$ and $\left.B_{\mathrm{c} 2}(0)\right)$ in Table I together with calculated by using Eq. 1 values for $\xi(0)$.

Skoskiewicz [23] reported that $\mathrm{PdH}_{\mathrm{x}}$ superconductors have the ratio:

$$
\alpha=\frac{2 \cdot \Delta(0)}{k_{B} \cdot T_{C}}=3.7
$$

where $\Delta(0)$ is ground state superconducting energy gap, $k_{\mathrm{B}}$ is the Boltzmann constant, which is very close to BCS weak-coupling limit of 3.53 [7]. Sansores et al. [51] performed the analysis of experimental electronic specific heat jump at $T_{\mathrm{c}}, \Delta C / C$, and confirmed the weakcoupling pairing strength in $\mathrm{PdH}_{\mathrm{x}}$ and $\mathrm{PdD}_{\mathrm{x}}$ by reporting:

$$
\frac{\Delta \mathrm{C}}{C}=1.44-1.59
$$

which is very close to BCS weak-coupling limit of 1.43 [7]. Thus, we use $\alpha$ value reported by Skoskiewicz (Eq. 8) [23] in our calculations below.

Bambakidis et al. [52] reported that $\mathrm{PdD}_{\mathrm{x}}$ compounds have charge carrier effective mass of:

$$
m_{e f f}^{*}=0.49 \cdot m_{e}
$$

where $m_{\mathrm{e}}$ is electron mass, which we use in calculations below for $\mathrm{PdH}_{\mathrm{x}}$ superconductors.

By using Eqs. 9-19 we calculate value which presented in Table I and Fig. 1. 
Table I. Deduced parameters for $\mathrm{PdH}_{\mathrm{x}}$ superconductors based on data reported by Balbaa and Manchester [50]. We assumed that $\frac{2 \cdot \Delta(0)}{k_{B} \cdot T_{c}}=3.7$ [23] and electron effective mass is $m_{e f f}^{*}=0.49 \cdot m_{e}$ [52]. Maximal and minimal $T_{\mathrm{c}} / T_{\mathrm{F}}$ values are in red bold.

\begin{tabular}{|c|c|c|c|c|c|c|c|c|c|}
\hline$x=\mathrm{H} / \mathrm{Pd}$ & $T_{\mathrm{c}}(\mathrm{K})$ & $\begin{array}{c}B_{\mathrm{c} 2}(0) \\
(\mathrm{mT})\end{array}$ & $\begin{array}{c}\text { Deduced } \\
\xi(0)(\mathrm{nm})\end{array}$ & $\begin{array}{c}v_{\mathrm{F}}\left(10^{5}\right. \\
\mathrm{m} / \mathrm{s})\end{array}$ & $\begin{array}{c}\Delta(0) \\
\mathrm{meV}\end{array}$ & $\begin{array}{c}\varepsilon_{\mathrm{F}} \\
\mathrm{meV}\end{array}$ & $\Delta(0) / \varepsilon_{\mathrm{F}}$ & $T_{\mathrm{F}}$ & $T_{\mathrm{c}} T_{\mathrm{F}}$ \\
\hline 0.821 & 1.488 & 39.5 & 91.3 & 1.03 & 0.237 & 14.9 & 0.016 & 173 & 0.009 \\
\hline 0.826 & 1.600 & 40.0 & 90.8 & 1.11 & 0.255 & 15.0 & 0.015 & 198 & $\mathbf{0 . 0 0 8}$ \\
\hline 0.843 & 2.061 & 68.0 & 69.6 & 1.09 & 0.329 & 16.6 & 0.020 & 193 & 0.011 \\
\hline 0.852 & 2.365 & 72.0 & 67.6 & 1.22 & 0.377 & 20.6 & 0.018 & 239 & 0.010 \\
\hline 0.862 & 2.672 & 96.5 & 58.4 & 1.19 & 0.426 & 19.6 & 0.022 & 228 & $\mathbf{0 . 0 1 2}$ \\
\hline 0.863 & 2.695 & 100.0 & 57.4 & 1.18 & 0.430 & 19.3 & 0.022 & 224 & $\mathbf{0 . 0 1 2}$ \\
\hline 0.875 & 3.090 & 111.0 & 54.5 & 1.28 & 0.493 & 22.9 & 0.022 & 265 & $\mathbf{0 . 0 1 2}$ \\
\hline 0.881 & 3.305 & 120.0 & 52.4 & 1.32 & 0.527 & 24.2 & 0.022 & 281 & $\mathbf{0 . 0 1 2}$ \\
\hline 0.887 & 3.590 & 125.0 & 51.3 & 1.40 & 0.572 & 27.4 & 0.021 & 317 & 0.011 \\
\hline 0.905 & 4.158 & 129.0 & 50.5 & 1.60 & 0.663 & 35.6 & 0.019 & 413 & 0.010 \\
\hline
\end{tabular}

It can be seen (Fig. 1) that $\mathrm{PdH}_{\mathrm{x}}$ falls just next to heavy fermion superconductors and thus these hydrogen-rich superconductors should be classified as unconventional superconductors.

\section{Deduced $B_{c 2}(0)$ and $\xi(0)$ for compressed LaH10}

In Fig. 2,a we show raw $B_{\mathrm{c} 2}(T)$ data for compressed $\mathrm{LaH}_{10}$ measured at the "cooling" stage (see for details Ref. 21). $B_{\mathrm{c} 2}(T)$ dataset was deduced from $R(T)$ curves showed in Fig. 2(a) of Drozdov et al [21] by using $50 \%$ of the normal state resistance criterion (raw data are given in Supplementary Table I). We note that Drozdov et al [21] also used this criterion to deduce $B_{\mathrm{c} 2}(T)$ and they also fitted data to Eq. 5 in their Fig. 2(b). In our Fig. 2, a we show fits to Eqs. 3-5,7 with deduced values collected in Table II.

For so-called "warming" stage, Drozdov et al [21] registered different $R(T)$ curves (Fig. 2(a) [21]) which we process in the same way and fit to Eqs. 3-5,7 (Fig. 2,b) (raw data are given in Supplementary Table II). Deduced $B_{\mathrm{c} 2}(0)$ and $\xi(0)$ values are collected in Table II. 
a

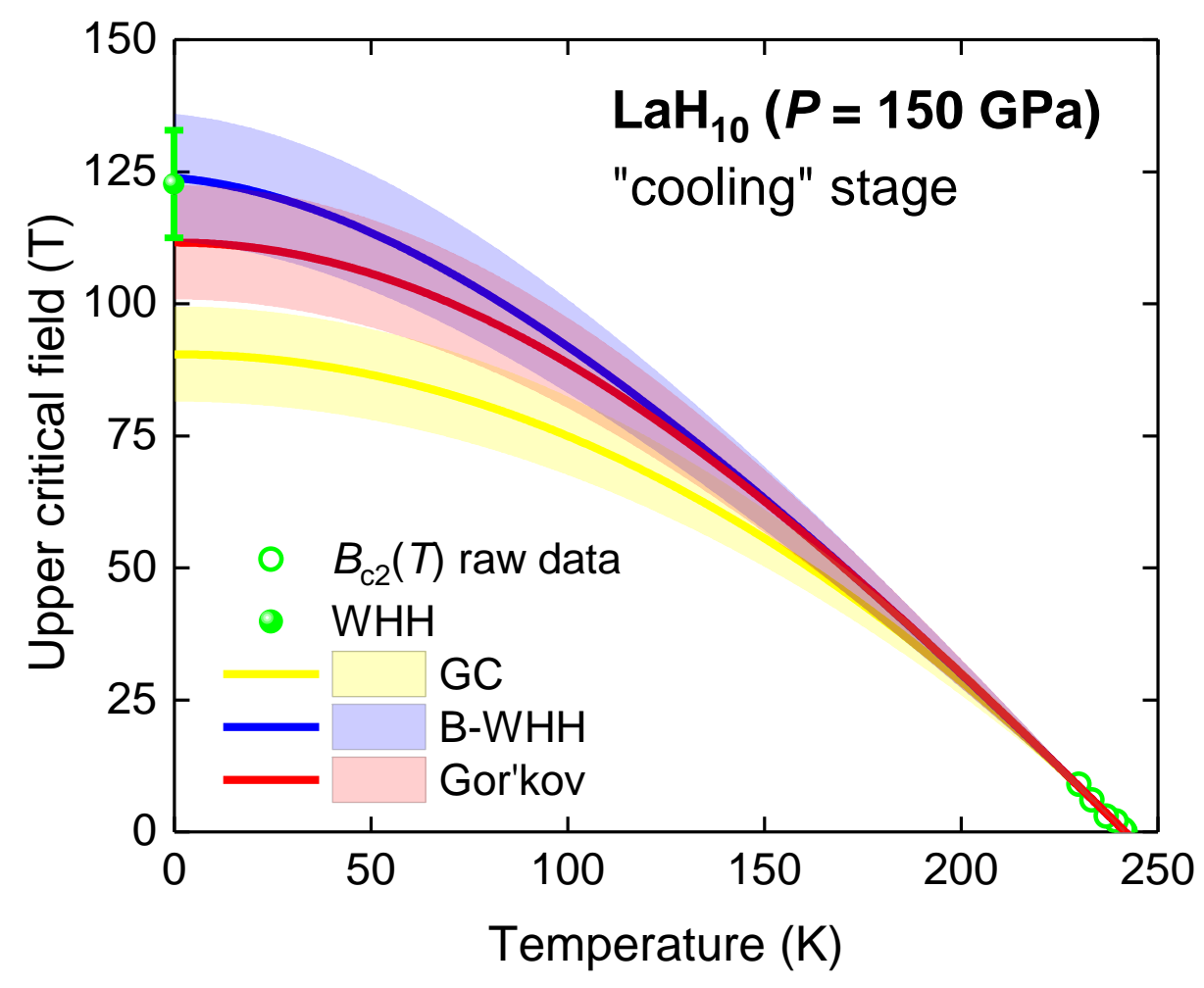

b

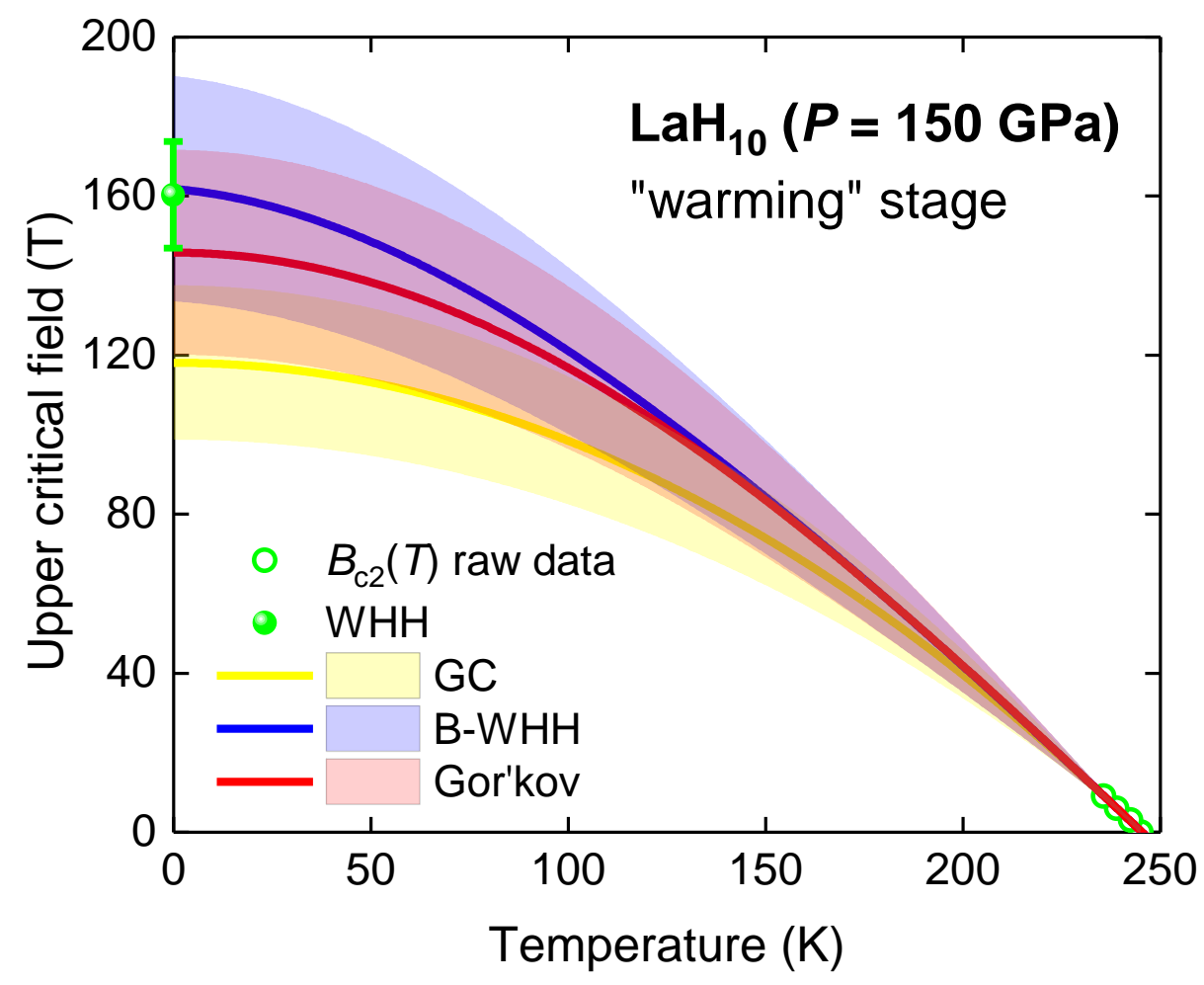

Figure 2. Superconducting upper critical field, $B_{\mathrm{c} 2}(T)$, data (magenta circles) and fits to four different model (Eqs. 3-5,7) for $\mathrm{LaH}_{10}$ compressed at pressure $P=150 \mathrm{GPa}$ (raw data are from Ref. 21) at (a) "cooling" and (b) "warming" stages. 95\% confidence bars are shown. 


\section{Compressed LaH10 in Uemura plot}

Kruglov et al. [33] performed first principles calculations and came to conclusion that compressed $\mathrm{LaH}_{10}$ is strong coupled superconductor with the ratio:

$$
\alpha=\frac{2 \cdot \Delta(0)}{k_{B} \cdot T_{C}}=5.00-5.55
$$

where $\Delta(0)$ is ground state superconducting energy gap, $k_{\mathrm{B}}$ is the Boltzmann constant.

Despite a fact that the upper boundary for $\alpha$ is the highest ever reported for electron-phonon mediated superconductors [9] we use this value as maximal $\alpha$ amplitude in our calculations below (Table II and Fig. 1).

We note that first principles calculations performed for $\mathrm{H}_{3} \mathrm{~S}$ also showed high values for the ratio (references can be found elsewhere $[4,9]$ ):

$$
\alpha=\frac{2 \cdot \Delta(0)}{k_{B} \cdot T_{C}}=4.5-4.7
$$

Table II. Deduced parameters for $\mathrm{LaH}_{10}$ superconductor subjected to external pressure of $P=150$ GPa. We assumed that electron effective mass is $m_{e f f}^{*}=3.0 \cdot m_{e}$ [58]. Maximal and minimal $T_{\mathrm{c}} / T_{\mathrm{F}}$

\begin{tabular}{|c|c|c|c|c|c|c|c|c|c|c|}
\hline Stage & Model & $\begin{array}{c}\text { Deduced } \\
T_{\mathrm{c}}(\mathrm{K})\end{array}$ & $\begin{array}{l}\text { Deduced } \\
\xi(0)(\mathrm{nm})\end{array}$ & $\begin{array}{l}\text { Assumed } \\
\frac{2 \cdot \Delta(0)}{k_{B} \cdot T_{C}}\end{array}$ & $\begin{array}{c}v_{\mathrm{F}}\left(10^{5}\right. \\
\mathrm{m} / \mathrm{s})\end{array}$ & $\begin{array}{l}\Delta(0) \\
\mathrm{meV}\end{array}$ & $\begin{array}{l}\varepsilon_{\mathrm{F}} \\
\mathrm{eV}\end{array}$ & $\Delta(0) / \varepsilon_{\mathrm{F}}$ & $\begin{array}{c}T_{\mathrm{F}}\left(10^{3}\right. \\
\mathrm{K})\end{array}$ & $T_{\mathrm{c}} / T_{\mathrm{F}}$ \\
\hline \multirow{6}{*}{ cooling } & \multirow[t]{2}{*}{ B-WHH } & \multirow{2}{*}{$\begin{array}{c}241.7 \pm \\
0.2\end{array}$} & \multirow{2}{*}{$\begin{array}{c}1.63 \pm \\
0.03\end{array}$} & 3.53 & $2.9 \pm 0.1$ & $\begin{array}{c}36.8 \pm \\
0.1\end{array}$ & $\begin{array}{c}0.70 \pm \\
0.03\end{array}$ & $\begin{array}{c}0.053 \pm \\
0.002\end{array}$ & $\begin{array}{c}8.1 \pm \\
0.3\end{array}$ & $\begin{array}{c}0.030 \pm \\
0.001\end{array}$ \\
\hline & & & & 5.55 & $4.5 \pm 0.1$ & $\begin{array}{c}57.8 \pm \\
0.1\end{array}$ & $\begin{array}{c}1.72 \pm \\
0.06\end{array}$ & $\begin{array}{c}0.034 \pm \\
0.001\end{array}$ & $\begin{array}{c}20.0 \pm \\
0.8\end{array}$ & $\begin{array}{c}0.012 \pm \\
0.001\end{array}$ \\
\hline & \multirow[b]{2}{*}{ GC } & \multirow{2}{*}{$\begin{array}{c}241.6 \pm \\
0.2\end{array}$} & \multirow{2}{*}{$\begin{array}{c}1.91 \pm \\
0.04\end{array}$} & 3.53 & $3.4 \pm 0.1$ & $\begin{array}{c}36.7 \pm \\
0.1\end{array}$ & $\begin{array}{c}0.96 \pm \\
0.04\end{array}$ & $\begin{array}{c}0.038 \pm \\
0.002\end{array}$ & $\begin{array}{c}11.1 \pm \\
0.5\end{array}$ & $\begin{array}{c}0.022 \pm \\
0.001\end{array}$ \\
\hline & & & & 5.55 & $5.3 \pm 0.1$ & $\begin{array}{c}57.8 \pm \\
0.1\end{array}$ & $\begin{array}{c}2.40 \pm \\
0.10\end{array}$ & $\begin{array}{c}0.024 \pm \\
0.002\end{array}$ & $\begin{array}{c}27.5 \pm \\
0.8\end{array}$ & $\begin{array}{c}0.009 \pm \\
0.001\end{array}$ \\
\hline & \multirow[t]{2}{*}{$\mathrm{G}$} & \multirow{2}{*}{$\begin{array}{c}241.7 \pm \\
0.2\end{array}$} & \multirow{2}{*}{$\begin{array}{c}1.72 \pm \\
0.04\end{array}$} & 3.53 & $3.0 \pm 0.1$ & $\begin{array}{c}36.8 \pm \\
0.1\end{array}$ & $\begin{array}{c}0.78 \pm \\
0.04\end{array}$ & $\begin{array}{c}0.047 \pm \\
0.003\end{array}$ & $\begin{array}{c}9.0 \pm \\
0.4\end{array}$ & $\begin{array}{c}0.027 \pm \\
0.001\end{array}$ \\
\hline & & & & 5.55 & $4.7 \pm 0.1$ & $\begin{array}{c}57.8 \pm \\
0.1\end{array}$ & $\begin{array}{c}1.92 \pm \\
0.09\end{array}$ & $\begin{array}{c}0.030 \pm \\
0.002\end{array}$ & $\begin{array}{c}22.3 \pm \\
1.1\end{array}$ & $\begin{array}{c}0.011 \pm \\
0.001\end{array}$ \\
\hline \multirow{6}{*}{ warming } & \multirow[t]{2}{*}{ B-WHH } & \multirow{2}{*}{$\begin{array}{c}245.3 \pm \\
0.2\end{array}$} & \multirow{2}{*}{$\begin{array}{c}1.43 \pm \\
0.03\end{array}$} & 3.53 & $2.6 \pm 0.1$ & $\begin{array}{c}37.3 \pm \\
0.1\end{array}$ & $\begin{array}{c}0.55 \pm \\
0.02\end{array}$ & $\begin{array}{c}0.068 \pm \\
0.003\end{array}$ & $\begin{array}{c}6.4 \pm \\
0.3\end{array}$ & $\begin{array}{c}0.038 \pm \\
0.002\end{array}$ \\
\hline & & & & 5.55 & $4.0 \pm 0.1$ & $\begin{array}{c}58.7 \pm \\
0.1\end{array}$ & $\begin{array}{c}1.37 \pm \\
0.06\end{array}$ & $\begin{array}{c}0.043 \pm \\
0.002\end{array}$ & $\begin{array}{c}15.9 \pm \\
0.7\end{array}$ & $\begin{array}{c}0.016 \pm \\
0.001\end{array}$ \\
\hline & \multirow[b]{2}{*}{ GC } & \multirow{2}{*}{$\begin{array}{c}245.3 \pm \\
0.2\end{array}$} & \multirow{2}{*}{$\begin{array}{c}1.67 \pm \\
0.03\end{array}$} & 3.53 & $3.0 \pm 0.1$ & $\begin{array}{c}37.3 \pm \\
0.1\end{array}$ & $\begin{array}{c}0.75 \pm \\
0.03\end{array}$ & $\begin{array}{c}0.050 \pm \\
0.003\end{array}$ & $\begin{array}{c}8.8 \pm \\
0.4\end{array}$ & $\begin{array}{c}0.028 \pm \\
0.001\end{array}$ \\
\hline & & & & 5.55 & $4.7 \pm 0.1$ & $\begin{array}{c}58.7 \pm \\
0.1\end{array}$ & $\begin{array}{c}1.86 \pm \\
0.09\end{array}$ & $\begin{array}{c}0.032 \pm \\
0.002\end{array}$ & $\begin{array}{c}21.6 \pm \\
0.9\end{array}$ & $\begin{array}{c}0.011 \pm \\
0.001\end{array}$ \\
\hline & \multirow[b]{2}{*}{ G } & \multirow{2}{*}{$\begin{array}{c}245.3 \pm \\
0.3\end{array}$} & \multirow{2}{*}{$\begin{array}{c}1.50 \pm \\
0.03\end{array}$} & 3.53 & $2.7 \pm 0.1$ & $\begin{array}{c}37.3 \pm \\
0.1\end{array}$ & $\begin{array}{c}0.61 \pm \\
0.03\end{array}$ & $\begin{array}{c}0.061 \pm \\
0.004\end{array}$ & $\begin{array}{c}7.1 \pm \\
0.4\end{array}$ & $\begin{array}{c}0.035 \pm \\
0.002\end{array}$ \\
\hline & & & & 5.55 & $4.2 \pm 0.1$ & $\begin{array}{c}58.7 \pm \\
0.1\end{array}$ & $\begin{array}{c}1.50 \pm \\
0.06\end{array}$ & $\begin{array}{c}0.039 \pm \\
0.003\end{array}$ & $\begin{array}{c}17.5 \pm \\
0.7\end{array}$ & $\begin{array}{c}0.014 \pm \\
0.001\end{array}$ \\
\hline
\end{tabular}
values are in red bold. 
From other hand, Hirsch and Marsiglio [53], Souza and Marsiglio [54], Harshman and Fiory [55], Kaplan and Imry [56] proposed different models for the superconductivity in $\mathrm{H}_{3} \mathrm{~S}$ either within classical BCS approach [56], either based on new concepts, and, for instance, Kaplan and Imry [56] showed that their model gives $\alpha$ within weak-coupling BCS limit:

$$
\alpha=\frac{2 \cdot \Delta(0)}{k_{B} \cdot T_{C}}=3.53
$$

This $\alpha$ value is in a good agreement with ones we deduced from experimental $B_{\mathrm{c} 2}(T)$ [14] and the self-field critical current density, $J_{c}(\mathrm{sf}, T)$, data [57]. Assuming that both hydrogen-rich counterparts $\left(\mathrm{H}_{3} \mathrm{~S}\right.$ and $\left.\mathrm{LaH}_{10}\right)$ have the same origin for near-room-temperature superconductivity, we calculated parameters for the latter (Table II and Fig. 1) by using weak-coupling limit of BCS (Eq. 25) as the lowest value for $\alpha$.

Kostrzewa et al. [58] reported that at $P=150 \mathrm{GPa}$ compressed $\mathrm{LaH}_{10}$ has charge carrier effective mass of:

$$
m_{e f f}^{*}=(2.9-3.2) \cdot m_{e} \approx 3.0 \cdot m_{e}
$$

where $m_{\mathrm{e}}$ is electron mass. This value is very close to $m_{e f f}^{*}=2.76 \cdot m_{e}$ calculated for compressed $\mathrm{H}_{3} \mathrm{~S}$ by Durajski [11]. For the simplicity, in our calculations below, for compressed $\mathrm{LaH}_{10}$ we use rounded value of $m_{e f f}^{*}=3.0 \cdot m_{e}$.

We perform the same calculation routine to one as we did for $\mathrm{PdH}_{\mathrm{x}}$ and calculated values are given in Table II and displayed in Fig. 1. Deduced $T_{\mathrm{c}} / T_{\mathrm{F}}$ values are in the range of $0.9 \cdot 10^{-2}<T_{C} / T_{F}<4.0 \cdot 10^{-2}$. It can be seen (Fig. 1) that $\mathrm{LaH}_{10}$ falls just above another hydrogen-rich counterpart, $\mathrm{H}_{3} \mathrm{~S}$, and both near-room-temperature superconductors are placed in unconventional superconductors band together with heavy fermions, $\mathrm{PdH}_{\mathrm{x}}$, fullerenes, pnictides and cuprates. 
More evidently our primary conclusion that $\mathrm{H}_{3} \mathrm{~S}$ and $\mathrm{LaH}_{10}$ are unconventional superconductors can be understood if one substitutes Eqs. 9-19 in the equation Eq. 1 and obtains:

$$
B_{c 2}\left(\frac{T}{T_{c}}=0\right)=\frac{\pi \cdot \phi_{0} \cdot k_{B}}{16 \cdot \hbar^{2}} \cdot m_{e f f}^{*} \cdot \alpha^{2} \cdot\left(\frac{T_{c}}{T_{F}}\right) \cdot T_{c}
$$

If $\mathrm{LaH}_{10}$ will be a conventional superconductor with $T_{c} / T_{F}=8.4 \cdot 10^{-6}$ (this is the ratio of electron-phonon mediated $\mathrm{Al}[18])$, then in accordance with Eq. 27:

$$
B_{c 2}\left(\frac{T}{T_{c}}=0\right)=70 m T
$$

If $T_{c} / T_{F}=7.4 \cdot 10^{-4}$ (which is the ratio of electron-phonon mediated $\mathrm{Nb}$ [18]), then:

$$
B_{c 2}\left(\frac{T}{T_{c}}=0\right)=6.2 T
$$

We note, that experimental $B_{\mathrm{c} 2}(T)$ value for $\mathrm{LaH}_{10}[21]$ is:

$$
B_{c 2}\left(\frac{T}{T_{c}}=0.96\right)=9.0 T
$$

Thus, Eqs. 27-30 show that despite a fact that currently $B_{\mathrm{c} 2}(T)$ dataset for $\mathrm{LaH}_{10}$ is available only at temperatures near $T_{\mathrm{c}}$ (i.e., $0.96 \leq \frac{T}{T_{c}} \leq 1.0$ ), this dataset is already enough to make a conclusion that $\mathrm{LaH}_{10}$ is unconventional superconductor.

Similar calculations for $\mathrm{H}_{3} \mathrm{~S}$ (based on experimental [15,16] and deduced [14] values) are:

$$
\begin{array}{ll}
B_{c 2}\left(\frac{T}{T_{c}}=0\right)<45 m T & \left(\mathrm{H}_{3} \mathrm{~S} \text { for } T_{c} / T_{F}=8.4 \cdot 10^{-6}\right) \\
B_{c 2}\left(\frac{T}{T_{c}}=0\right)<3.9 T & \left(\mathrm{H}_{3} \mathrm{~S} \text { for } T_{c} / T_{F}=7.4 \cdot 10^{-4}\right) .
\end{array}
$$

These values (if even we will make comparison of ones with very strictly defined $B_{\mathrm{c} 2}(T)$ (at $R$ $=0 \Omega$ ) and pressure of $P=160 \mathrm{GPa}$ (which is shifted from optimal pressure of $P=155 \mathrm{GPa}$ )) are at least in one order of magnitude lower than experimental data:

$$
B_{c 2}\left(\frac{T}{T_{c}} \approx \frac{1}{3}\right)=65 T \quad\left(\mathrm{H}_{3} \mathrm{~S} \text { at } P=160 \mathrm{GPa}\right) .
$$


Eqs. 27-33 show that both near-room-temperature superconductors, $\mathrm{H}_{3} \mathrm{~S}$ and $\mathrm{LaH}_{10}$, cannot be classified as conventional superconductors, despite a fact that astonishing Ashcroft's prediction $[59,60]$ about near-room-temperature superconductivity in hydrogen-rich compounds was based on BCS theory.

In overall, all extrapolated $B_{\mathrm{c} 2}(0)$ values for $\mathrm{LaH}_{10}$ (Fig. 2) are well below the Pauli depairing field of:

$$
B_{p}(0)=\frac{2 \cdot \Delta(0)}{g \cdot \mu_{B}}=\alpha \cdot \frac{k_{B} \cdot T_{c}}{g \cdot \mu_{B}}=630-1,000 T \gg B_{c 2}(0)
$$

where $g=2$ and $\mu_{B}=\frac{e \cdot \hbar}{2 \cdot m_{e}}$ is the Bohr magneton. According to Gor'kov's note [61], Eq. 34 means that the mean-free path, $l$, of the electrons is large compared with the coherence length, $\xi(0)$ :

$$
l \gg \xi(T)>\xi(0) \sim 1.4-1.9 \mathrm{~nm}
$$

However, the lower limit for the mean-free path, $l$, is only in a few times exceeded the lattice constants for any structural unit cell of $\mathrm{LaH}_{10}$ either proposed by first principles calculations [33], either deduced by the fits of experimental data to Fm-3m symmetry $(a=0.51019(5) \mathrm{nm}$ [21]) or $P 4 / \mathrm{nmm}$ symmetry $(a=0.37258(6) \mathrm{nm}, \mathrm{c}=0.50953(12) \mathrm{nm}$ [21]). This means that charge carrier scattering is large in $\mathrm{LaH}_{10}$.

\section{Thermodynamic fluctuations in compressed $\mathrm{LaH}_{10}$}

In our previous papers $[57,62]$ we answered a question about possible limitation of $T_{\mathrm{c}}$ in compressed $\mathrm{H}_{3} \mathrm{~S}$ by the thermodynamic fluctuations of the order parameter. There are phase [63] and amplitude [64] fluctuations of the order parameter in superconductors. These fluctuations for the case of three dimensional (3D) superconductors (like, $\mathrm{Th}_{4} \mathrm{H}_{15}, \mathrm{PdH}_{\mathrm{x}}, \mathrm{H}_{3} \mathrm{~S}$ and $\mathrm{LaH}_{10}$ ) have characteristic temperatures:

$$
T_{\text {fluc,phase }}=\frac{0.55 \cdot \phi_{0}^{2}}{\pi^{3 / 2} \cdot \mu_{0} \cdot k_{B}} \cdot \frac{1}{\kappa^{2} \cdot \xi(0)}
$$




$$
T_{f l u c, a m p}=\frac{\phi_{0}^{2}}{12 \cdot \pi \cdot \mu_{0} \cdot k_{B}} \cdot \frac{1}{\kappa^{2} \cdot \xi(0)}
$$

where $\kappa=\lambda(0) / \xi(0)$ is the Ginzburg-Landau parameter, and $\lambda(0)$ is the ground state London penetration depth.

Due to for $T h_{4} \mathrm{H}_{15}$ and $\mathrm{Th}_{4} \mathrm{D}_{15}$ phases the Ginzburg-Landau parameter is unknown we do not consider this interesting case herein.

For $\mathrm{PdH}_{\mathrm{x}}$ system, $\kappa$ was found to be $\kappa=1.2-2.0$ [50], and calculated values for $T_{\text {fluc,phase }}$ and $T_{\text {fluc,amp }}$ show that thermodynamic fluctuations for superconductors in palladiumhydrogen system are negligible low (Table III).

In our recent paper [14], we deduced $\xi(0)$ in $\mathrm{H}_{3} \mathrm{~S}$ more accurately in comparison with values we used to calculate $T_{f l u c, p h a s e}$ and $T_{f l u c, a m p}$ in our previous papers $[57,62]$. By taking in account that $\mathrm{H}_{3} \mathrm{~S}$ exhibits $\kappa=88-105$ [57,62,65], we revisited $T_{\text {fluc,phase }}$ and $T_{\text {fluc,amp }}$ at two applied pressures of $P=155 \mathrm{GPa}$ and $160 \mathrm{GPa}$ (Table III). At each pressure, we used maximal and minimal deduced $\xi(0)$ values to show the range of variation for $T_{\text {fluc,phase }}$ and $T_{\text {fluc,amp. }}$

To calculate $T_{\text {fluc,phase }}$ and $T_{\text {fluc,amp }}$ (Eqs. 36,37) for compressed $\mathrm{LaH}_{10}$ there is a need to make an assumption about the value for Ginzburg-Landau parameter, $\kappa$. Our approach is based on an assumption that $\kappa$ for $\mathrm{LaH}_{10}$ will be not much different from $\kappa$ for $\mathrm{H}_{3} \mathrm{~S}$ and other unconventional superconductors (primarily, pnictides and cuprates) which have values within a range of $\kappa=60-120$ [65-71]. Calculated $T_{\text {fluc,phase }}$ and $T_{\text {fluc,amp }}$ for $\mathrm{LaH}_{10}$ are given in Table III.

Examination of the values in Table III leaded us to an important finding that both nearroom-temperature superconductors have very large fluctuations of the order parameter amplitude, which, in some scenarios, have characteristic temperatures, $T_{\text {fluc,amp, }}$, of only about $15 \%$ above observed transition temperature, $T_{\mathrm{c}}$. This is similar to cuprates and pnictides 
which have large fluctuations of order parameter phase $[57,62]$ (which causes the suppression of the observed $T_{\mathrm{c}}$ by about $30 \%$ from its mean-field value, $\left.T_{c}^{\text {mean-field }}[57,62]\right)$.

Table III. Calculated fluctuation temperatures for $\mathrm{H}_{3} \mathrm{~S}$ (at $P=155$ and $160 \mathrm{GPa}$ ) and $\mathrm{LaH}_{10}$ $(P=150 \mathrm{GPa})$. Assumed electron effective masses are $m_{e f f}^{*}=2.76 \cdot m_{e}[11]$ for $\mathrm{H}_{3} \mathrm{~S}$ and $m_{e f f}^{*}=3.0 \cdot m_{e}$ for $\mathrm{LaH}_{10}[50]$. The largest ratios for $\frac{T_{c}}{T_{\text {fluc,phase }}}$ and $\frac{T_{c}}{T_{\text {fluc,amp }}}$ are marked in red bold. Deduced $T_{\mathrm{c}}$ and $\xi(0)$ values for $\mathrm{H}_{3} \mathrm{~S}$ are from Ref. 14.

\begin{tabular}{|c|c|c|c|c|c|c|c|c|c|}
\hline Material & Stage & $\begin{array}{c}\text { Pressure } \\
(\mathrm{GPa})\end{array}$ & $\begin{array}{c}\text { Deduced } \\
T_{\mathrm{c}}(\mathrm{K})\end{array}$ & $\begin{array}{l}\text { Deduced } \\
\xi(0)(\mathrm{nm})\end{array}$ & $\begin{array}{c}\text { Assumed } \\
\kappa\end{array}$ & $\begin{array}{c}T_{\text {fluc,phase }} \\
\text { (K) }\end{array}$ & $\begin{array}{c}T_{\text {fluc,amp }} \\
\text { (K) }\end{array}$ & $T_{\mathrm{c}} / T_{\text {fluc,phase }}$ & $T_{\mathrm{c}} / T_{\text {fluc,amp }}$ \\
\hline $\mathrm{PdH}_{\mathrm{x}}$ & N/ A & N/A & 4.16 & 50.5 & 2 & $1.2 \cdot 10^{5}$ & $3.2 \cdot 10^{4}$ & $3.5 \cdot 10^{-5}$ & $1.3 \cdot 10^{-4}$ \\
\hline \multirow{9}{*}{$\mathrm{H}_{3} \mathrm{~S}$} & \multirow{9}{*}{ N/A } & \multirow{4}{*}{155} & \multirow{2}{*}{$182 \pm 1$} & \multirow{2}{*}{$\begin{array}{c}1.97 \pm \\
0.02\end{array}$} & 88 & $1590 \pm 20$ & $428 \pm 5$ & $0.114 \pm 0.001$ & $0.43 \pm 0.01$ \\
\hline & & & & & 105 & $1120 \pm 11$ & $304 \pm 5$ & $0.163 \pm 0.002$ & $0.61 \pm 0.01$ \\
\hline & & & \multirow{2}{*}{$189 \pm 1$} & \multirow{2}{*}{$\begin{array}{c}1.68 \pm \\
0.01\end{array}$} & 88 & $1870 \pm 10$ & $502 \pm 3$ & $0.101 \pm 0.001$ & $0.38 \pm 0.01$ \\
\hline & & & & & 105 & $1313 \pm 11$ & $353 \pm 5$ & $0.144 \pm 0.001$ & $0.54 \pm 0.01$ \\
\hline & & \multirow{5}{*}{160} & \multirow{3}{*}{$150 \pm 3$} & \multirow{3}{*}{$\begin{array}{c}2.67 \pm \\
0.05\end{array}$} & 88 & $1176 \pm 23$ & $316 \pm 6$ & $0.128 \pm 0.003$ & $0.48 \pm 0.01$ \\
\hline & & & & & & & & & \\
\hline & & & & & 105 & $826 \pm 16$ & $222 \pm 4$ & $0.182 \pm 0.004$ & $0.68 \pm 0.02$ \\
\hline & & & \multirow{2}{*}{$172 \pm 2$} & \multirow{2}{*}{$\begin{array}{c}2.06 \pm \\
0.01\end{array}$} & 88 & $1524 \pm 8$ & $410 \pm 2$ & $0.113 \pm 0.001$ & $0.42 \pm 0.01$ \\
\hline & & & & & 105 & $1071 \pm 5$ & $288 \pm 2$ & $0.161 \pm 0.002$ & $0.60 \pm 0.01$ \\
\hline \multirow{4}{*}{$\mathrm{LaH}_{10}$} & \multirow[t]{2}{*}{ cooling } & \multirow[t]{2}{*}{150} & \multirow{2}{*}{$\begin{array}{c}241.7 \pm \\
0.2\end{array}$} & \multirow{2}{*}{$\begin{array}{c}1.63 \pm \\
0.03\end{array}$} & 60 & $4150 \pm 70$ & $\begin{array}{c}1113 \pm \\
30\end{array}$ & $0.058 \pm 0.001$ & $0.21 \pm 0.01$ \\
\hline & & & & & 120 & $1036 \pm 20$ & $278 \pm 6$ & $0.233 \pm 0.004$ & $0.87 \pm 0.02$ \\
\hline & \multirow[t]{2}{*}{ warming } & \multirow[t]{2}{*}{150} & \multirow{2}{*}{$\begin{array}{c}245.3 \pm \\
0.2\end{array}$} & \multirow{2}{*}{$\begin{array}{c}1.43 \pm \\
0.03\end{array}$} & 60 & $4720 \pm 100$ & $\begin{array}{c}1270 \pm \\
30\end{array}$ & $0.052 \pm 0.001$ & $0.19 \pm 0.01$ \\
\hline & & & & & 120 & $1180 \pm 25$ & $317 \pm 7$ & $0.208 \pm 0.005$ & $0.77 \pm 0.02$ \\
\hline
\end{tabular}

\section{Self-field critical currents in compressed $\mathrm{LaH}_{10}$}

We need to stress, that there is fundamental limit to obtain answers on many important questions in regards of $\mathrm{H}_{3} \mathrm{~S}$ and $\mathrm{LaH}_{10}$ by performing the upper critical field studies, because experimentally available magnetic fields, $B_{\text {appl }}$, even, if top world facilities will be in use $[15,16,31]$, are too low for these materials. Thus, there is a need to find different experimental techniques to reveal the nature of superconductivity in these and, perhaps, many others near-room-temperature hydrogen-based superconductors. One possible way to perform 
this is to study temperature dependent self-field critical currents, $I_{\mathrm{c}}(\mathrm{sf}, T)[37,65]$, from which several fundamental parameters of the superconductor, i.e., the ground state superconducting energy gap, $\Delta(0)$, the ground state London penetration depth, $\lambda(0)$, and relative jump in the specific heat at $T_{\mathrm{c}}, \Delta C / C$, can be deduced. We already showed that this approach works for compressed $\mathrm{H}_{3} \mathrm{~S}$ [57] by performing analysis of the self-field magnetization critical current densities reported by Drozdov et al. [1].

We note that experimental technique to perform critical current measurements in ultrahigh-pressure diamond cells is under developing for about twenty years [72-75] and fundamental possibility to measure $I_{\mathrm{c}}(\mathrm{sf}, T)$ in $\mathrm{LaH}_{10}$ has been already demonstrated by Somayazulu et al [20] in their Fig. 4 and Supplementary Information. However, $I_{\mathrm{c}}(\mathrm{sf}, T)$ measurement techniques (and particularly inside of ultrahigh-pressure diamond cells) need to be further developed, because these measurements require great precaution due to the danger of sample "burning" during transport current pulse [76-78], and $I_{\mathrm{c}}(\mathrm{sf}, T)$ data collecting is still state-of-art $[79,80]$. For instance, $\mathrm{LaH}_{10}$ samples degradation, after $I_{\mathrm{c}}(\mathrm{sf}, T)$ measurements reported by Somayazulu et al [20], is more likely originated from the sample "burning" under transport current flow. In this regard, recently registered effect [81] of the linearization of surface magnetic field, $B_{\text {surf }}(I)$, vs transport current rise at the onset of power dissipation can be considered as a new option. We showed recently, that this effect $[81,82]$ works when external magnetic field, $B_{\text {appl }}$, is applied to the sample $[83,84]$.

There are also two powerful optical spectroscopy techniques, one is the Raman spectroscopy (which is under on-going developing to be used in ultrahigh-pressure diamond cells for last decades [85-88]), and another is the angle resolved photoemission spectroscopy (ARPES) which was recently advanced [89] to be applicable to study superconducting films under transport current flow. 


\section{Conclusions}

In this paper we analyse experimental $B_{\mathrm{c} 2}(T)$ data of near-room-temperature superconductor, $\mathrm{LaH}_{10}$ (recently reported by Drozdov et al. [21]), of palladium-hydrogen superconductors, $\mathrm{PdH}_{\mathrm{x}}$ (reported by Balbaa and Manchester [50]) and of $\mathrm{Th}_{4} \mathrm{H}_{15}-\mathrm{Th}_{4} \mathrm{D}_{15}$ (reported by Satterthwaite and Toepke [22]). We come to conclusion that all discovered to date hydrogen-rich superconductors for which fundamental superconducting parameters beyond $T_{\mathrm{c}}$ were measured (in this list we do not include $\mathrm{NbTiH}_{\mathrm{x}}, \mathrm{PtH}_{\mathrm{x}}, \mathrm{SiH}_{4}$ and $\mathrm{PH}_{3}$ for which only experimental $T_{\mathrm{c}}$ vs pressure are known), i.e., $\mathrm{Th}_{4} \mathrm{H}_{15}-\mathrm{Th}_{4} \mathrm{D}_{15}, \mathrm{PdH}_{\mathrm{x}}, \mathrm{H}_{3} \mathrm{~S}$ and $\mathrm{LaH}_{10}$, are unconventional superconductors.

In addition, we find that both near-room-temperature superconductors, $\mathrm{H}_{3} \mathrm{~S}$ and $\mathrm{LaH}_{10}$, are subjected by strong thermodynamic fluctuations of the order parameter amplitude. We note, that this is very similar to pnictides and cuprates in which superconducting state is affected by strong fluctuations of the order parameter phase.

\section{Acknowledgement}

Author thanks Prof. V. E. Antonov (Institute of Solid State Physics, Russian Academy of Sciences) and Prof. E. Gregoryanz (University of Edinburgh) for valuable discussions. Author also thanks financial support provided by the state assignment of Minobrnauki of Russia (theme "Pressure" No. AAAA-A18-118020190104-3) and by Act 211 Government of the Russian Federation, contract No. 02.A03.21.0006.

\section{References}

[1] Drozdov A P, Eremets M I, Troyan I A, Ksenofontov V, Shylin S I 2015 Conventional superconductivity at 203 kelvin at high pressures in the sulfur hydride system Nature $\mathbf{5 2 5} 73$ 76

[2] Bednorz J G and Mueller K A 1986 Possible high $T_{\mathrm{c}}$ superconductivity in the Ba-La-CuO system Z. Phys. B 64 189-193 
[3] Eremets M I and Drozdov A P 2016 High-temperature conventional superconductivity Phys.-Usp. 59 1154-1160

[4] Gor'kov L P and Kresin V Z 2018 Colloquium: High pressure and road to room temperature superconductivity Rev. Mod. Phys. 90011001

[5] Pickett W and Eremets M I 2019 The quest for room-temperature superconductivity in hydrides Physics today $\mathbf{7 2}$ 52-58

[6] Flores-Livas J A, Boeri L, Sanna A, Profeta G, Arita R, Eremets M 2019 A perspective on conventional high-temperature superconductors at high pressure: Methods and materials arXiv: 1905.06693

[7] Bardeen J, Cooper L N, Schrieffer J R 1957 Theory of superconductivity Phys. Rev. 108, 1175-1204

[8] Mazin I I 2015 Extraordinarily conventional Nature 525 40-41

[9] Nicol E J and Carbotte J P 2015 Comparison of pressurized sulfur hydride with conventional superconductors Phys. Rev. B 91 220507(R)

[10] Errea I et al 2015 High-pressure hydrogen sulfide from first principles: A strongly anharmonic phonon-mediated superconductor Phys. Rev. Lett. 114157004

[11] Durajski A P 2016 Quantitative analysis of nonadiabatic effects in dense $\mathrm{H}_{3} \mathrm{~S}$ and $\mathrm{PH}_{3}$ superconductors Scientific Reports 638570

[12] Jarlborg T and Bianconi A 2016 Breakdown of the Migdal approximation at Lifshitz transitions with giant zero-point motion in the $\mathrm{H}_{3} \mathrm{~S}$ superconductor Scientific Reports 624816 [13] Durajski A P and Szczęśniak R 2018 Structural, electronic, vibrational, and superconducting properties of hydrogenated chlorine J. Chem. Phys. 149074101 [14] Talantsev E F 2019 Classifying superconductivity in compressed $\mathrm{H}_{3} \mathrm{~S}$ Modern Physics Letters B 331950195

[15] Mozaffari S, et al 2019 Superconducting hydride under extreme field and pressure Los Alamos National Laboratory Report LA-UR-18-30460, doi: 10.2172/1481108.

[16] Mozaffari S, et al 2019 Superconducting phase-diagram of $\mathrm{H}_{3} \mathrm{~S}$ under high magnetic fields Nature Communications 102522

[17] Uemura Y J, et al 1989 Universal correlations between $T_{\mathrm{c}}$ and $\frac{n_{s}}{m^{*}}$ (carrier density over effective mass) in high- $T_{\mathrm{c}}$ cuprate Phys. Rev. Lett. 62 2317-2320

[18] Uemura Y J 2004 Condensation, excitation, pairing, and superfluid density in high- $T_{\mathrm{c}}$ superconductors: the magnetic resonance mode as a roton analogue and a possible spinmediated pairing J. Phys.: Condens. Matter 16 S4515-S4540

[19] Hirsch J E, Maple M B, Marsiglio F 2015 Superconducting materials classes:

Introduction and overview Physica C 514 1-8

[20] Somayazulu M, Ahart M, Mishra A K, Geballe Z M, Baldini M, Meng Y, Struzhkin V V and R. J. Hemley R J 2019 Evidence for superconductivity above $260 \mathrm{~K}$ in lanthanum superhydride at megabar pressures Phys. Rev. Lett. 122027001

[21] Drozdov A P, et al 2019 Superconductivity at $250 \mathrm{~K}$ in lanthanum hydride under high pressures Nature $\mathbf{5 6 9}$ 528-531

[22] Satterthwaite C B and Toepke I L 1970 Superconductivity of hydrides and deuterides of thorium Phys. Rev. Lett. 25 741-743

[23] Skoskiewicz T 1973 Superconductivity in the palladium-hydrogen system Phys. Stat.

Sol. (b) 59329

[24] Eremets M I, Trojan I A, Medvedev S A, Tse J S, Yao Y 2008 Superconductivity in hydrogen dominant materials: Silane Science 319 1506-1509

[25] Drozdov A P, Eremets M I, Troyan I A 2015 Superconductivity above $100 \mathrm{~K}$ in $\mathrm{PH}_{3}$ at high pressures arXiv: 1508.06224

[26] Matsuoka T, et al. 2019 Superconductivity of platinum hydride Phys Rev B 99144511 
[27] Antonov V E, Belash I T, Zakharov M S, Orlov V A, Rashupkin V I 1986 Int. J. Hydrogen Energy 11475.

[28] Ginzburg V L and Landau L D 1950 On the theory of superconductivity Zh. Eksp. Teor. Fiz. 20 1064-1082

[29] Helfand E and Werthamer N R 1966 Temperature and purity dependence of the superconducting critical field, $H_{\mathrm{c} 2}$. II. Phys. Rev. 147 288-294

[30] Werthamer N R, Helfand E and Hohenberg P C 1966 Temperature and purity dependence of the superconducting critical field, $H_{\mathrm{c} 2}$. III. Electron spin and spin-orbit effects Phys. Rev. 147 295-302

[31] Hänisch J, et al. 2015 High field superconducting properties of $\mathrm{Ba}\left(\mathrm{Fe}_{1-\mathrm{x}} \mathrm{Co}_{\mathrm{x}}\right)_{2} \mathrm{As}_{2}$ thin films Sci. Rep. 517363

[32] Liu H, Naumov I I, Hoffmann R, Ashcroft N W and Hemley R J 2017 Potential high- $T_{\mathrm{c}}$ superconducting lanthanum and yttrium hydrides at high pressure Proc. Natl. Acad. Sci. 114 6990-6995

[33] Kruglov I A, et al. 2019 Superconductivity of $\mathrm{LaH}_{10}$ and $\mathrm{LaH}_{16}$ : new twists of the story arXiv:1810.01113

[34] Baumgartner T, Eisterer M, Weber H W, Fluekiger R, Scheuerlein C, Bottura L 2014

Effects of neutron irradiation on pinning force scaling in state-of-the-art $\mathrm{Nb}_{3} \mathrm{Sn}$ wires

Supercond. Sci. Technol. 27015005

[35] Gorter C J and H. Casimir H 1934 On supraconductivity I Physica 1 306-320

[36] Poole P P, Farach H A, Creswick R J, Prozorov R 2007 Superconductivity (2-nd Edition, London, UK).

[37] Talantsev E F, Crump W P, Island J O, Xing Y, Sun Y, Wang J, J. L. Tallon J L 2017

2D Materials 4025072

[38] Pal B, et al. 2019 Experimental evidence of a very thin superconducting layer in epitaxial indium nitride Supercond. Sci. Technol. 32015009

[39] Gor'kov L P 1960 The critical supercooling field in superconductivity theory Soviet Physics JETP 10 593-599

[40] Jones C K, Hulm J K, Chandrasekhar B S 1964 Upper critical field of solid solution alloys of the transition elements Rev. Mod. Phys. 36 74-76

[41] Stritzker B and Buckel W 1972 Superconductivity in the palladium-hydrogen and the palladium-deuterium systems Zeitschrift für Physik A Hadrons and nuclei 257 1-8

[42] Yussouff M, Rao B K and Jena P 1995 Reverse isotope effect on the superconductivity of PdH, PdD, and PdT Solid State Communications 94 549-553

[43] Villa-Cortés S and Baquero R 2018 On the calculation of the inverse isotope effect in $\mathrm{PdH}(\mathrm{D})$ : A Migdal-Eliashberg theory approach Journal of Physics and Chemistry of Solids 119 80-84

[44] Ostanin S, Borisov V, Fedorov D V, Salamatov E I, Ernst A and Mertig I 2019 Role of tetrahedrally coordinated dopants in palladium hydrides on their superconductivity and inverse isotope effect Journal of Physics: Condensed Matter 31075703

[45] Caton R and Satterthwaite C B 1977 Preparation and characterization of massive $\mathrm{Th}_{4} \mathrm{H}_{15}$ and $\mathrm{Th}_{4} \mathrm{D}_{15}$ Journal of the Less Common Metals 52307

[46] Miller J F, Caton R H, Satterthwaite C B 1976 Low-temperature heat capacity of normal and superconducting thorium hydride and thorium deuteride. Phys. Rev. B 142795

[47] Ye J T, et al. 2012 Superconducting dome in a gate-tuned band insulator Science 338 1193

[48] Qian T, et al. 2011 Absence of a holelike Fermi surface for the iron-based $\mathrm{K}_{0.8} \mathrm{Fe}_{1.7} \mathrm{Se}_{2}$ superconductor revealed by angle-resolved photoemission spectroscopy Phys. Rev. Lett. 106 187001 
[49] Hashimoto K, Cho K, Shibauchi T, Kasahara S, Mizukami Y, Katsumata R, Tsuruhara Y, Terashima T, Ikeda H, Tanatar M A, Kitano H, Salovich N, Giannetta R W, Walmsley P, Carrington A, Prozorov R, Matsuda Y 2012 A sharp peak of the zero-temperature penetration depth at optimal composition in $\mathrm{BaFe}_{2}\left(\mathrm{As}_{1-\mathrm{x}} \mathrm{P}_{\mathrm{x}}\right)_{2}$ Science 336 1554-1557

[50] Balbaa I S and Manchester F D 1983 Superconductivity of $\mathrm{PdH}_{\mathrm{x}}$ in relation to its phase diagram: I. Magnetic measurements J. Phys. F: Met. Phys. 13 395-404

[51] Sansores L E, Taguena-Martinez J and Sanchez A M 1981 The specific heat and critical magnetic field of superconducting PdH(D) Journal of Low Temperature Physics 43 205-215 [52] Bambakidis G, Smith R J, and Otterson D A 1968 Electrical resistivity as a function of deuterium concentration in palladium NASA Report Number TN D-4970

[53] Hirsch J E and Marsiglio F 2015 Hole superconductivity in $\mathrm{H}_{2} \mathrm{~S}$ and other sulfides under high pressure Physica C $\mathbf{5 1 1}$ 45-49

[54] Souza T X R and Marsiglio F 2016 Systematic study of the superconducting critical temperature in two- and three-dimensional tight-binding models: A possible scenario for superconducting $\mathrm{H}_{3} \mathrm{~S}$ Phys. Rev. B 94184509

[55] Harshman D R and Fiory A T 2017 Compressed $\mathrm{H}_{3}$ S: inter-sublattice Coulomb coupling in a high- $T_{\mathrm{c}}$ superconductor J. Phys.: Condens. Matter 29445702

[56] Kaplan D and Imry Y 2018 High-temperature superconductivity using a model of hydrogen bonds Proc. Nat. Acad. Sci. 115 5709-5713

[57] Talantsev E F, Crump W P, Storey J G, Tallon J L 2017 London penetration depth and thermal fluctuations in the sulphur hydride $203 \mathrm{~K}$ superconductor Annalen der Physics $\mathbf{5 2 9}$ 1600390

[58] Kostrzewa M, Szczesniak K M, Durajski A P, Szczesniak R 2019 From $\mathrm{LaH}_{10}$ to roomtemperature superconductors arXiv:1905.12308

[59] Ashcroft N W 1968 Metallic hydrogen: A high-temperature superconductor? Phys. Rev.

Lett. 21 1748-1749

[60] Ashcroft N W 2004 Hydrogen dominant metallic alloys: High temperature superconductors? Phys. Rev. Lett. 92187002

[61] Gor'kov L P 1963 An estimate of the limiting values of the critical fields for hard superconductors Soviet Physics JETP 17518

[62] Tallon J L and Talantsev E F 2018 Compressed $\mathrm{H}_{3} \mathrm{~S}$, superfluid density and the quest for room-temperature superconductivity Journal of Superconductivity and Novel Magnetism 31 619-624

[63] Emery V J and Kivelson S A 1995 Importance of phase fluctuations in superconductors with small superfluid density Nature 374 434-437

[64] Bulaevskii L N, Ginzburg V L, Sobyanin A A 1988 Macroscopic theory of superconductors with small coherence length Physica C 152 378-388

[65] Talantsev E F, Crump W P, Tallon J L 2017 Thermodynamic parameters of single- or multi-band superconductors derived from self-field critical currents Annalen der Physics $\mathbf{5 2 9}$ 1700197

[66] Sakoda M, Iida K and Naito M 2018 Recent progress in thin-film growth of Fe-based superconductors: Superior superconductivity achieved by thin films Supercond. Sci. Technol. 31093001.

[67] Iida K, Hänisch J, and Tarantini C 2018 Fe-based superconducting thin films on metallic substrates: Growth, characteristics, and relevant properties Applied Physics Reviews 5031304.

[68] Eisterer M 2018 Radiation effects on iron-based superconductors Supercond. Sci. Technol. 31013001 
[69] Kauffmann-Weiss S et al. 2019 Microscopic origin of highly enhanced current carrying capabilities of thin $\mathrm{NdFeAs}(\mathrm{O}, \mathrm{F})$ films Nanoscale Advances 1147

https://doi.org/10.1039/C9NA00147F

[70] Hänisch J et al. 2019 Fe-based superconducting thin films - Preparation and tuning of superconducting properties Supercond. Sci. Technol. in press https://doi.org/10.1088/1361$\underline{6668 / \mathrm{ab} 1 \mathrm{c} 00}$

[71] Talantsev E F 2019 Evaluation of practical level of critical current densities in pnictides and recently discovered superconductors Supercond. Sci. Technol. 32084007

[72] Timofeev Y A, Struzhkin V V, Hemley R J, Mao H-K and Gregoryanz E A 2002 Improved techniques for measurement of superconductivity in diamond anvil cells by magnetic susceptibility Rev. Sci. Instrum. 73371

[73] Eremets M I, Struzhkin V V, Mao H-K, Hemley R J 2003 Exploring superconductivity in low-Z materials at megabar pressures Physica B: Condensed Matter 329-333 1312-1316

[74] Gavriliuk A G, Mironovich A A, and Struzhkin V V 2009 Miniature diamond anvil cell for broad range of high pressure measurements Rev. Sci. Instrum. 80043906

[75] Guo J, Wu Q, and Sun L 2018 Advanced high-pressure transport measurement system integrated with low temperature and magnetic field Chin. Phys. B 27077402

[76] Talantsev E F, Strickland N M, Hoefakker P, Xia J A, Long N J 2008 Critical current anisotropy for second generation HTS wires Current Applied Physics 8 388-390

[77] Strickland N M, Hoffmann C, Wimbush S C, Pooke D M, Huang T, Lazic Z, Chamritski V, Talantsev E F, Long N J and Tallon J L 2014 Cryogen-free 1kA-class $I_{\mathrm{c}}$ measurement system featuring an 8 T HTS magnet J. Phys. Conf. Ser. 507022037

[78] Lao M, Hänisch J, Kauffmann-Weiss S, Gehring R, Fillinger H, Drechsler A, Holzapfel B 2019 High current variable temperature electrical characterization system for superconducting wires and tapes with continuous sample rotation in a split coil magnet Review of Scientific Instruments 90015106

[79] Talantsev E F and Mataira R C 2018 Polar projections for big data analysis in applied superconductivity AIP Advances $\mathbf{8} 075213$

[80] Strickland N M, Hoffmann C, Wimbush S C 2014 Review of Scientific Instruments 85 113907

[81] Talantsev E F 2017 On the fundamental definition of critical current in superconductors arXiv: 1707.07395

[82] Talantsev E F, Strickland N M, Wimbush S C, Crump W P 2017 The onset of dissipation in high-temperature superconductors: Self-field experiments AIP Advances 7 125230

[83] Talantsev E F, Strickland N M, Wimbush S C, Brooks J, Pantoja A E, Badcock R A, Storey J G, Tallon J L 2018 The onset of dissipation in high-temperature superconductors: magnetic hysteresis and field dependence Scientific Reports 814463

[84] Talantsev E F and Brooks J 2019 The onset of dissipation in high-temperature superconductors: flux trap, hysteresis and in-field performance of multifilamentary $\mathrm{Bi}_{2} \mathrm{Sr}_{2} \mathrm{Ca}_{2} \mathrm{Cu}_{3} \mathrm{O}_{10+x}$ wires Mater. Res. Express 6026002

[85] Goncharov A F, Gregoryanz E, Mao H-K, Liu Z and Hemley R J 2000 Optical evidence for a nonmolecular phase of nitrogen above $150 \mathrm{GPa}$ Phys. Rev. Lett. 85 1262-1265

[86] Chen X-A, et al. 2008 Pressure-induced metallization of silane Proc. Natl. Acad. Sci. USA 105 20-23

[87] Yuan Y, et al. 2019 Stoichiometric evolutions of $\mathrm{PH}_{3}$ under high pressure: implication for high- $T_{\mathrm{c}}$ superconducting hydrides National Science Review 6 524-531

[88] Dalladay-Simpson P, et al. 2019 Band gap closure, incommensurability and molecular dissociation of dense chlorine Nature Communications 101134 
[89] Kaminski, A, Rosenkranz S, Norman M R, Randeria M, Li Z Z, Raffy H, and Campuzano J C 2016 Destroying coherence in high-temperature superconductors with current flow Phys. Rev. X 6031040 


\title{
SUPPLEMENTARY INFORMAION
}

\section{for}

\section{Classifying hydrogen-rich superconductors}

\author{
E. F. Talantsev ${ }^{1,2}$
}

${ }^{1}$ M.N. Miheev Institute of Metal Physics, Ural Branch, Russian Academy of Sciences, 18, S. Kovalevskoy St., Ekaterinburg, 620108, Russia

${ }^{2}$ NANOTECH Centre, Ural Federal University, 19 Mira St., Ekaterinburg, 620002, Russia

E-mail: evgeny.talantsev@imp.uran.ru

Supplementary Table I. $B_{\mathrm{c} 2}(T)$ dataset reported by Drozdov et al. [1] for "cooling" stage of $\mathrm{LaH}_{10}$ subjected to pressure of $P=150 \mathrm{GPa}$. Values were deduced by $50 \%$ of normal state resistance criterion from Fig. 2(a) [1].

\begin{tabular}{|c|c|}
\hline$T(\mathrm{~K})$ & $B_{\mathrm{c} 2}(\mathrm{~T})$ \\
\hline 241.68 & 0 \\
\hline 241.68 & 0 \\
\hline 241.53 & 0.2 \\
\hline 241.26 & 0.5 \\
\hline 239.41 & 2 \\
\hline 236.86 & 3 \\
\hline 236.91 & 3 \\
\hline 233.23 & 6 \\
\hline 229.99 & 9 \\
\hline
\end{tabular}

Supplementary Table II. $B_{\mathrm{c} 2}(T)$ dataset reported by Drozdov et al. [1] for "warming" stage of $\mathrm{LaH}_{10}$ subjected to pressure of $P=150 \mathrm{GPa}$. Values were deduced by $50 \%$ of normal state resistance criterion from Fig. 2(a) [1].

\begin{tabular}{|c|c|}
\hline$T(\mathrm{~K})$ & $B_{\mathrm{c} 2}(\mathrm{~T})$ \\
\hline 245.1 & 0 \\
\hline 242.5 & 3 \\
\hline 239.0 & 6 \\
\hline 235.7 & 9 \\
\hline
\end{tabular}

\section{Reference}

[1] Drozdov A P, et al 2019 Superconductivity at $250 \mathrm{~K}$ in lanthanum hydride under high pressures Nature $\mathbf{5 6 9} 528-531$ 